\title{
Biosynthesis of silver nanoparticles using Withania somnifera (L.) Dunal extract and its antibacterial activity against food pathogens
}

\author{
R. Jagadheeswari, T. Lakshmi*, Sri Renukadevi Balusamy*, Sheba David** and S. Raghunandha Kumar \\ Department of Pharmacology, Saveetha Dental College, Saveetha Institute of Medical and Technical Sciences (SIMATS), Saveetha University, \\ Chennai-602117, Tamil Nadu, India \\ *Department of Food Science and Biotechnology, Sejong University, 98 Gunja-dong, Gwangjin-gu, Seoul 143-747, Republic of Korea \\ **PAPRSB Institute of Health Sciences, Universiti Brunei Darussalam, Jalan Tungku Link, Bandar Seri Begawan BE1410, Brunei
}

\begin{tabular}{l} 
Article Info \\
\hline Article history \\
Received 9 April 2020 \\
Revised 27 May 2020 \\
Accepted 30 May 2020 \\
Published online 30 June 2020
\end{tabular}

Keywords

Nanoparticles

Biosynthesis

Toxicity

Antibacterial

Biomedical

\begin{abstract}
Silver nanoparticles play a significant role in the field of biology and medicine. It exerts high toxicity to various micro-organisms. The aim of the present study was to green synthesis silver nanoparticles using Withania somnifera (L.) Dunal (Ashwagandha) and evaluate its antibacterial activity against common food pathogens. W. somnifera belongs to Solanaceae (Nightshade) family. The biochemical constituent of ashwagandha plant is called withanolides. In the present study, the silver nanoparticles were synthesized in less than $30 \mathrm{~min}$, using the leaf extracts of the plant, W. somnifera and its antimicrobial activity against food pathogens was evaluated. The synthesized nanoparticles exhibited antibacterial activity against $E$. coli and B. subtilis which was evaluated by agar-well diffusion method. The silver nanoparticles synthesized via green route were highly toxic to multidrug resistant bacteria like Pseudomonas, Staphylococcus aureus, E. coli and due to its great potential, it may be used in the biomedical application in near future.
\end{abstract}

\section{Introduction}

Nanoparticles have enormous applications in science and technology. Effective nanoparticles commonly used today are those made from noble metals in particular Ag, Pt, Au and Pd (Veera babu Nagati et al., 2013). Compared to other nanoparticles, silver nanoparticles play a significant role in the field of biology and medicine. The nanoparticles can be synthesized by physical, chemical and biological methods but biological synthesis of silver nanoparticles have several advantages over physical and chemical methods as it is cheap, can be achieved with a single process and ecofriendly (Anal Jha and Prasad, 2010). In the biological method, living organisms such as bacteria, fungi and plants are used for the nanoparticles synthesis (Bankura and Mighty, 2012).

Silver nanoparticles are nanoparticles of silver between $1 \mathrm{~nm}$ and $100 \mathrm{~nm}$ in size. Three major sources of synthesizing silver nanoparticles were reported: bacteria, fungi, and plant extracts (Bhattacharya et al., 2001). Preparation of nanoparticles by biological ways require three major elements-solvent medium for synthesis, the environmentally friendly reducer, and a nontoxic useful agent (Bharani and Thirunethiran, 2012). Several infectious diseases caused by antibiotic-resistant infective microorganism has

\section{Corresponding author: Dr. T. Lakshmi}

Associate Profressor, Department of Pharmacology, Saveetha Dental College, Saveetha SIMATS, Saveetha University, Chennai-602117, Tamil Nadu, India E-mail: lakshmi@saveetha.com

Tel.: +91-9884299939

Copyright (C) 2020 Ukaaz Publications. All rights reserved.

Email: ukaaz@yahoo.com; Website: www.ukaazpublications.com brought the main focus back on the silver and its mixture forms. The properties of silver nanoparticles significant to human treatments are under investigation in laboratory and animal studies, assessing potential efficacy, toxicity, and costs (Das et al., 2010).

The plant Withania somnifera (L.) Dunal commonly known as Ashwagandha or Indian ginseng belongs to Solanaceae (Nightshade) family (Elsakka et al.,1990). It contains potentially active constituents of alkaloids-isopelletierine and anaferins (Pandey et al., 2018; Su Cheol Baek et al., 2019). The biochemical constituents of Ashwagandha plant are called withanolides (Elumalai et al., 2010). These are steroidal lactones- with anolides and with aferins, saponin containing an additional acyl groups (Iuvone et al., 2003). Root of Ashwagandha is used as an anti-inflammatory drug for swellings, tumours, scrofula and rheumatism (Gupta and Kaur, 2018) and as a sedative and hypnotic in anxiety neurosis (Fidel Martinez-Gutierrez and Peggy, 2010). Ashwagandha is believed as general energy-promoting and has disease prevention property and improves the immunity (Yong Song et al., 2009). In the present study, the silver nanoparticles were synthesized in less time, using the leaf extracts of the plant, $W$. somnifera and its antibacterial activity against food pathogens, E. coli and B. subtilis were evaluated using agar-well diffusion method.

\section{Materials and Methods}

\subsection{Preparation of the plant extract}

W. somnifera plant material was obtained as a sample from Life Care Phytolabs Private Limited. Leaves were collected and washed 3-4 times in distilled water. Then, it is dried in shade for 
7-14 days. The dried leaves are made into powder. $1 \mathrm{~g}$ of powder was dissolved in $100 \mathrm{ml}$ of distilled water and boiled for 5-10 min at $60-70^{\circ} \mathrm{C}$. The solution was filtered using Whatman's No 1 filter paper. Finally, it was collected and stored at $4^{\circ} \mathrm{C}$.

\subsection{Synthesis of nanoparticles}

The filtered plant extract and the $10 \mathrm{mM}$ of silver nitrate solution and mixed in a $250 \mathrm{ml}$ conical flask in 1:4 ratio. The solution was kept in magnetic stirrer with hot plate $\left(600^{\circ} \mathrm{C}\right)$ for $30 \mathrm{~min}$ for green synthesis of nanoparticle synthesis. The color change was observed visually. The supernatant mixture was centrifuged at 10,000 rpm for $15 \mathrm{~min}$ and the pellet was again washed in distilled water and the resultant pellet was air dried at dark condition for 2 days.

\subsection{Characterization of nanoparticles}

The synthesized nanoparticles were preliminary characterized using UV-visible spectroscopy. About $3 \mathrm{ml}$ of the solution was taken in curettes and scanned in double-beam UV-visible spectrophotometer from $300 \mathrm{~nm}$ to $700 \mathrm{~nm}$ wavelength. The results were recorded for the graphical analysis. Further, the nanoparticles were subjected to FT-IR and Scanning electron microscopy by following Venkatesan et al. (2014).

\subsection{Antibacterial activity}

The agar well-diffusion method was used to determine the antibacterial activity of silver NPs. Different concentrations of silver NPs were tested against E. coli, B. Subtilis. Fresh bacterial suspension $\left(10^{8} \mathrm{CFU} / \mathrm{ml}\right)$ was dispersed on the surface of MullerHinton agar plates. Different concentrations of NPs (20, 40, and 60 $\mu 1)$ were incorporated into the wells and the plates were incubated at $37^{\circ} \mathrm{C}$ for $24 \mathrm{~h}$. The antibiotics were used as positive control. Zone of inhibition was measured for each plate. The diameter of the clear zone was measured using a ruler.

\section{Results}

\subsection{Visual observation}

Figure 1 shows the visual change in the colour of the Ashwagandha plant extract from greenish colour to brown colour upon addition of the silver nitrate solution, which indicates the formation of the silver nanoparticles.
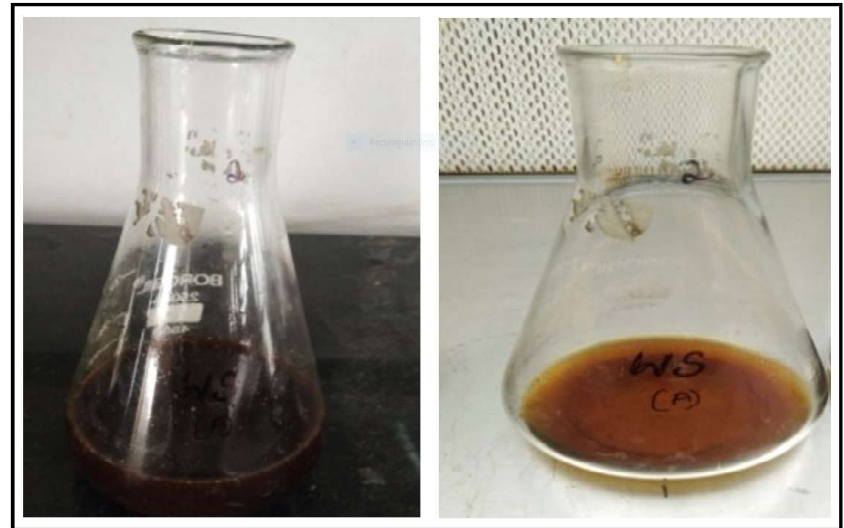

Figure 1: Synthesis of silver Nanoparticles: Visual observation of the change in the reaction mixture from greenish to brown colour upon addition of silver nitrate solution.

\subsection{UV-vis spectroscopy}

The presence of nanoparticles was confirmed by obtaining a spectrum in the visible range of 300-700 $\mathrm{nm}$. The absorption spectrum of the synthesized silver nanoparticles is shown in the Figure 2. The results revealed a peak at prominent peak at $420 \mathrm{~nm}$, which confirmed the formation of silver nanoparticles.

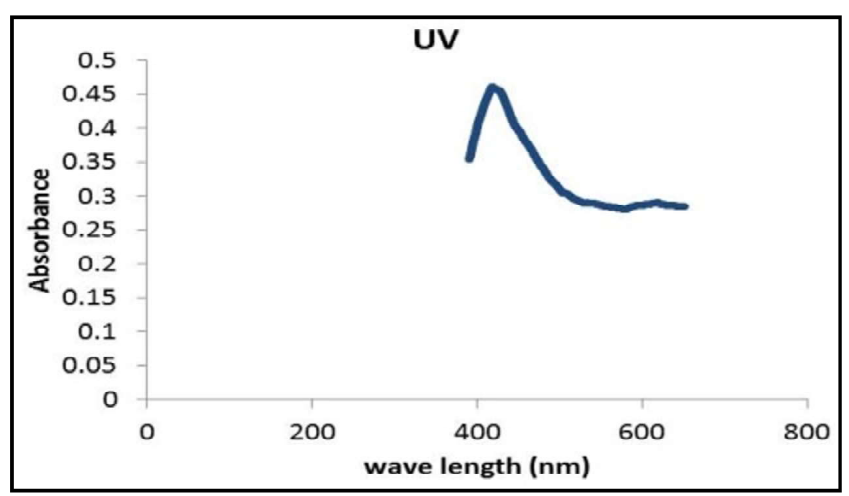

Figure 2: UV-vis spectroscopy: A prominent peak is observed at $420 \mathrm{~nm}$, indicating the silver nanoparticles.

\subsection{FT-IR analysis}

FT-IR analysis is generally used to analyse the chemical adsorption and functional groups in the newly synthesized nanoparticles. Figure 3 shows the FT-IR transmission spectra of the Ashwagandha extract mediated silver nanoparticles. The FT-IR analysis has revealed the characteristic peaks of the silver nanoparticles.

\subsection{FE-SEM analysis}

Figure 4 shows the FE-SEM analysis of the silver nanoparticles, which provides a clear idea regarding the shape and size distribution of the synthesized nanoparticles. It is clearly evident from the FESEM micrographs that nanoparticles are evenly distributed without formation of clumps.

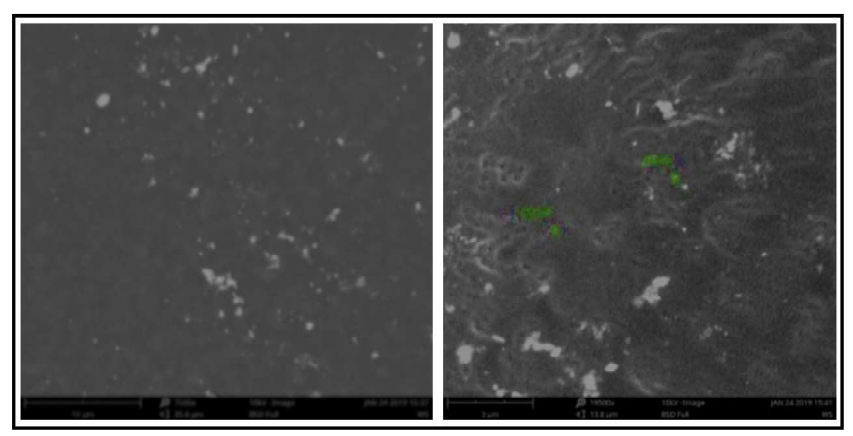

Figure 4: FE-SEM analysis of silver nanoparticles.

\subsection{Antibacterial effect of silver nanoparticles}

The antibacterial analysis of the synthesized silver nanoparticles are analysed by performing the agar-well diffusion experiment. Figure 5 shows the bar graph and the culture plates representing the zone of inhibition of the food pathogenic bacteria (E. coli, B. subtilis) upon treatment with the silver nanoparticles. The results revealed that the nanoparticles created a zone of $2.2 \mathrm{~mm}$ and 2.9 $\mathrm{mm}$ for E.coli and B. subtilis, respectively. 


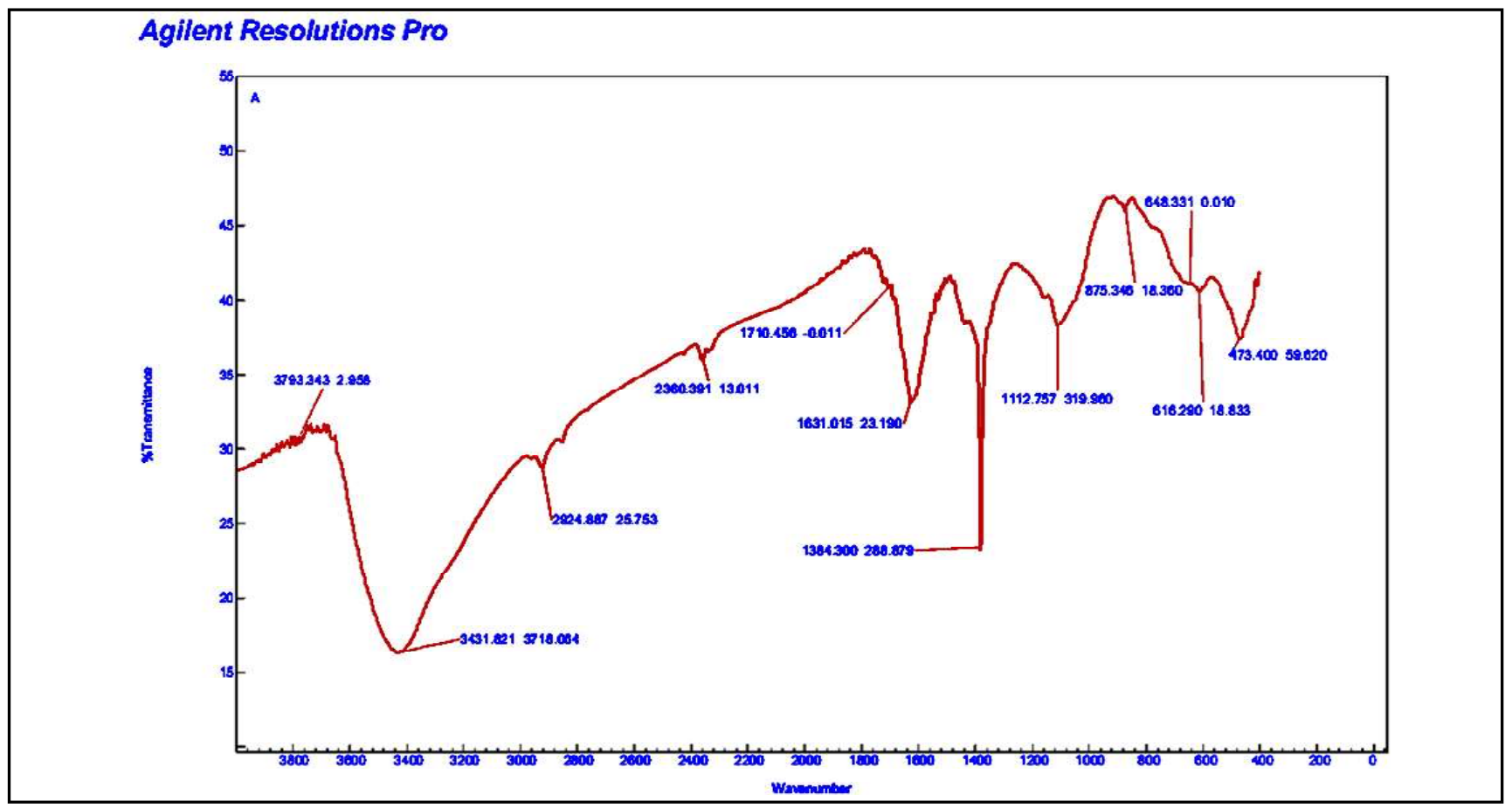

Figure 3: FT-IR analysis of the silver nanoparticles.

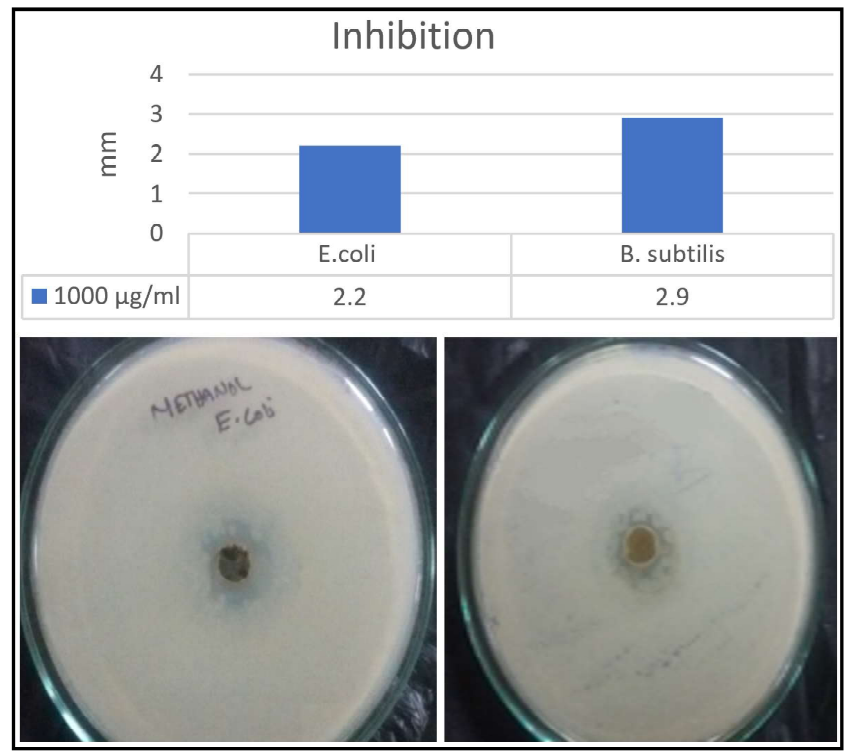

Figure 5: Antibacterial effect of silver nanoparticles: Bar graph and the representative images of culture plates of agar-well diffusion experiment for analysing the effect of silver nanoparticles on E.coli and B. subtilis.

\section{Discussion}

Green synthesis of nanoparticles has attained much importance due to its ease of synthesis and environmentally safe nature (Rajeshkumar and Bharath, 2017). In the present study, to synthesise plant mediated silver nanoparticles, Ashwagandha extract and silver nitrate solution were taken in 1:4 ratio and the colour changed from green to brown, which indicated the formation of silver nanoparticles. The change in this colour is due to the reduction of the silver ion (Rajeshkumar and Bharath, 2017). This indicated the preliminary confirmation for the formation of Ashwagandha silver nanoparticles (Justin Packia Jacob et al., 2012). The reduction of pure Ag+ ions was monitored by measuring the UV-Vis spectrum of the reaction medium after $30 \mathrm{~min}$.

Silver nanoparticles exhibit reddish brown colour in aqueous solution due to excitation of surface plasmon vibrations in silver nanoparticles. The maximum absorbance spectrum of the silver nanoparticles was observed at $420 \mathrm{~nm}$ (Geethika et al., 2018). Further, the results of the FE-SEM analysis revealed the nanoparticles are fairly distributed, which indicated the successful synthesis process (Kim Soo-Hwan and Hyeong-Seon Lee, 2011; Vaidyanathan et al., 2010). The FE-SEM micrograph clearly showed the particle size range of our nanoparticles to be within $86 \mathrm{~nm}$ and high density silver nanoparticles synthesized in the presence of Ashwagandha leaf extract was further confirmed by the development of silver nanostructures (Vidhi Mehrotra et al., 2011).

The nanoparticles were further subjected to FT-IR analysis which revealed the characteristic peaks of the silver nanoparticles as mentioned in the previous studies (Ziauddin et al., 1996; Sushma Jain et al., 2001). Further, to analyse the effect of these nanoparticles to inhibit the food pathogens, E. coli and B. subtilis; agar-well diffusion method was performed as mentioned in previous studies (Sukumaran et al., 2011; Shankar et al., 2003). It was clear from the results that the silver nanoparticles effectively inhibit the growth of $E$. coli and B. subtilis, but the effect was much prominent on $B$. subtilis compared to that of E. coli (Vankar and Shukla, 2012).

\section{Conclusion}

This study demonstrated the potential of leaf extract of the Ashwagandha plant in reducing aqueous $\mathrm{Ag}+$ to $\mathrm{Ag} 0$ ions and formation 
of ecofriendly silver nanoparticles with fairly well-defined dimensions. It provides evidence that the leaves are good source for synthesizing stable silver nanoparticles in lesser time. These ecofriendly nanoparticles could be used as an excellent source against multidrug resistant bacteria, enhancing the wound healing process that can also act as anticancer, antistress agent. It can also be used in large-scale for synthesizing nanoparticles from other inorganic materials. The silver nanoparticles synthesized via green route are highly toxic to multidrug resistant bacteria and due to its great potential, it may be used in the biomedical application in near future.

\section{Conflict of interest}

The authors declare that there are no conflicts of interest in the course of conducting the research. All the authors had final decision regarding the manuscript and decision to submit the findings for publication.

\section{References}

Anal K. Jha and K. Prasad (2010). Green synthesis of silver nanoparticles using cycas leaf. Int. J. Green Nanotech. Phys. Chem., 1:110-117.

Ae Yong Song, A. and Beom Soo Kim (2009). Rapid biological synthesis of silver nanoparticles using plant leaf extracts. Bioprocess Biosyst. Eng., 32:7984.

Bankura K.P. and D. Maity (2012). Synthesis, characterization and antimicrobial activity of dextran stabilized silver nanoparticles in aqueous medium. Carbohyd. Polym., 89:1159-1165.

Bharani, M. and Thirunethiran Karpagam (2012). Synthesis and characterization of silver nano particles from wrightia tinctoria. Int. J. Appl. Bio. PharmTech., 3:58-63.

Bhattacharya, A.; Ghosal, S. and Bhattacharya, S.K. (2001). Antioxidant effect of Withania somnifera glycol withanolides in chronic footshock stress induced perturbations of oxidative free radical scavenging enzymes and lipid peroxidation in rat frontal cortex and striatum. J. Ethnopharmacol., 74:1-6.

Das, R.; Nath, S.S.; Chakdar, D.; Gope, G. and Bhattacharjee, R. (2010). Synthesis of silver nanoparticles and their optical properties. J. Exp. Nanosci., 5(4)357-362.

Elsakka, M.; Grigorescu, E. and Stanescu, U. (1990). New data referring to chemistry of Withania somnifera species. Rev. Med. Chir. Soc. Med. Nat. Iasi., 94:385-387.

Elumalai, E.K.; T.N.V.K.V. Prasad and V. Kambala (2010). Green synthesis of silver nanoparticle using Euphorbia hirta L. and their antifungal activities, Arch. Appl. Sci. Res., 2(6):76-81.

Fidel Martinez-Gutierrez and Peggy L. Olive (2010). Synthesis characterization and evaluation of antimicrobial and cytotoxic effect of silver and titanium nanoparticles. Nanomed. Nanotechol. Bio. Med., 6:681-688.

Geethika, B.; Sameer, S.S.; Vishal, L.A. and Thangavelu, L. (2018). Green synthesis of silver nanoparticles from heartwood extracts-family of fabaceae. Drug Inv. Today, 10:2018.

Gupta, M. and Kaur, G. (2018). Withania somnifera as a potential anxiolytic and anti-inflammatory candidate against systemic lipopolysaccharideinduced neuroinflammation. Neuromol. Med., 20:343-362. https:/ /doi.org/10.1007/s12017-018-8497-7
Iuvone, T.; Esposito, G.; Capasso, F. and Izzo, A. (2003). Induction of nitric oxide synthase expression by Withania somnifera in macrophages. Life Sci., 72:1617-1625.

Justin Packia Jacob, S.; J.S. Finub and Anand Narayanan (2012). Synthesis of silver nanoparticles using Piper longum leaf extracts and its cytotoxic activity against Hep-2 cell line. Colloids and Surfaces B: Biointerfaces, 91:212-214.

Kim, Soo-Hwan and Hyeong-Seon Lee (2011). Antibacterial activity of silvernanoparticles against staphylococcus aureus and E. coli. Korean J. Microbiol. Biotechnol., 39(1):77-85.

Pandey, S.S.; Singh, S. and Pandey, H. (2018). Endophytes of Withania somnifera modulate in planta content and the site of withanolide biosynthesis. Sci. Rep., 8:5450. https://doi.org/10.1038/s41598018-23716-5

Vankar, P.S. and Shukla, D. (2012). Biosynthesis of silver nanoparticles using lemon leaves extract and its applications for antimicrobial finish on fabric. Appl. Nanosci., 2:163-168.

Rajeshkumar, S. and Bharath, L.V. (2017). Mechanism of plant-mediated synthesis of silver nanoparticles: A review on biomolecules involved, characterisation and antibacterial activity. Chem. Biol. Interact., 273:219-227.

Shankar, S.S.; Ahmed, A. and Sastry, M. (2003). Geranium leaf assisted biosynthesis of silver nanoparticles, Biotechnology Progress, 19:1627-1631.

Su Cheol Baek; Seoyoung Lee; Sil Kim; Mun Seok Jo; Jae Sik Yu; Yoon-Joo Ko; Young-Chang Cho and Ki Hyun Kim (2019). Withaninsams A and B: Phenylpropanoid esters from the roots of Indian ginseng (Withania somnifera). Plants, 8(12):527. https://doi.org/10.3390/plants8120527

Sushma Jain; Sunil Dutt Shukla; Kanika Sharma and Maheep Bhatnagar (2001). Neuroprotective effects of Withania somnifera Dunn. in hippocampal sub-regions of female albino Rat. Phytother Res., 15:544-548.

Sukumaran, S.; Kiruba, S.; Mahesh, M.; Nisha, S.R.; Miller, P.Z. and Ben, C.P. (2011). Phytochemical constituents and antibacterial efficacy of the flowers of Peltophorum pterocarpum (DC) Baker ex Heyne. Asian Pac. J. Trop. Med., 4(9):735-738.

Vaidyanathan, R.; Gopalram, S.; Kalishwaralal, K.; Deepak, V.; Pandian, S.R.K. and Gurunathan, S. (2010). Enhanced silver nanoparticle synthesis by optimization of nitrate reductase activity. Colloids Surf. B., 75(1):335-341.

Veera Babu Nagati and Rama Koyyati (2013). Green Synthesis of plantmediated silver nanoparticles using Withania somnifera leaf extract and evaluation of their antimicrobial activity. Int. J. Adv. Res., 1(9):307-313.

Vidhi Mehrotra; Shubhi Mehrotra;Vandna Kirar; Radhey Shyam; Kshipra Misra; Ashwani Kumar Srivastava and Shoma Paul Nandi (2011). Antioxidant and antimicrobial activities of aqueous extract of Withania somnifera against methicillin-resistant Staphylococcus aureus. J. Microbiol. Biotech. Res., 1(1):40-45.

Ziauddin, M.; Phansalkar, N.; Patki, P.; Sham Diwanay and Bhushan Patwardhan (1996). Studies on the immunomodulatory effects of Ashwagandha. J. Ethnopharmacol., 50:69-76.

Citation: R. Jagadheeswari, T. Lakshmi, Sri Renukadevi Balusamy, Sheba David and S. Raghunandha Kumar (2020). Biosynthesis of silver nanoparticles using Withania somnifera extract and its anti-bacterial activity against food pathogens. Ann. Phytomed., 9(1):195-198. http://dx.doi.org/10.21276/ap.2020.9.1.25 